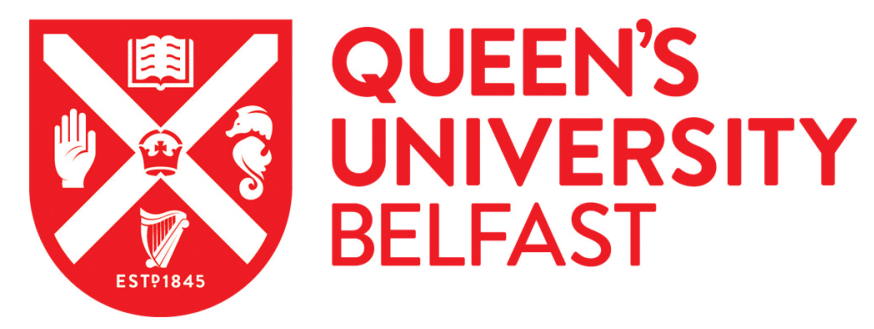

\title{
Temporal Narrowing of Neutrons Produced by High-Intensity Short- Pulse Lasers
}

Higginson, D. P., Vassura, L., Gugiu, M. M., Antici, P., Borghesi, M., Brauckmann, S., Diouf, C., Green, A., Palumbo, L., Petrascu, H., Sofia, S., Stardubtsev, M., Willi, O., Kar, S., Negoita, F., \& Fuchs, J. (2015). Temporal Narrowing of Neutrons Produced by High-Intensity Short-Pulse Lasers. Physical Review Letters, 115(5), [054802]. https://doi.org/10.1103/PhysRevLett.115.054802

Published in:

Physical Review Letters

Document Version:

Publisher's PDF, also known as Version of record

Queen's University Belfast - Research Portal:

Link to publication record in Queen's University Belfast Research Portal

Publisher rights

@ 2015 American Physical Society

\section{General rights}

Copyright for the publications made accessible via the Queen's University Belfast Research Portal is retained by the author(s) and / or other copyright owners and it is a condition of accessing these publications that users recognise and abide by the legal requirements associated with these rights.

Take down policy

The Research Portal is Queen's institutional repository that provides access to Queen's research output. Every effort has been made to ensure that content in the Research Portal does not infringe any person's rights, or applicable UK laws. If you discover content in the Research Portal that you believe breaches copyright or violates any law, please contact openaccess@qub.ac.uk. 


\title{
Temporal Narrowing of Neutrons Produced by High-Intensity Short-Pulse Lasers
}

\author{
D. P. Higginson, ${ }^{1,10}$ L. Vassura, ${ }^{1,2}$ M. M. Gugiu, ${ }^{3}$ P. Antici, ${ }^{2,4,5}$ M. Borghesi, ${ }^{6,7}$ S. Brauckmann,${ }^{8}$ C. Diouf, ${ }^{1}$ \\ A. Green, ${ }^{6}$ L. Palumbo, ${ }^{2}$ H. Petrascu, ${ }^{3}$ S. Sofia, ${ }^{1}$ M. Stardubtsev, ${ }^{9}$ O. Willi, ${ }^{8}$ S. Kar, ${ }^{6}$ F. Negoita, ${ }^{3}$ and J. Fuchs ${ }^{1,9}$ \\ ${ }^{1}$ Laboratoire pour l'Utilisation des Lasers Intenses, UMR 7605 CNRS-CEA-École Polytechnique-Université Paris VI, \\ 91128 Palaiseau, France \\ ${ }^{2}$ Dipartimento SBAI, Università di Roma “La Sapienza,”Via Scarpa 14-16, 00161 Roma, Italy \\ ${ }^{3}$ IFIN-HH, 077125 Bucharest-Magurele, P.O. Box MG-6, Romania \\ ${ }^{4}$ INRS-EMT, Varennes, Québec J3X 1S2, Canada \\ ${ }^{5}$ Istituto Nazionale di Fisica Nucleare, Via E. Fermi, 40-00044 Frascati, Italy \\ ${ }^{6}$ School of Mathematics and Physics, The Queen's University of Belfast, Belfast BT7 1NN, United Kingdom \\ ${ }^{7}$ Institute of Physics of the ASCR, ELI-Beamlines project, Na Slovance 2, 18221 Prague, Czech Republic \\ ${ }^{8}$ Institut für Laser und Plasmaphysik, Heinrich Heine Universität Düsseldorf, D-40225 Düsseldorf, Germany \\ ${ }^{9}$ Institute of Applied Physics, 46 Ulyanov Street, 603950 Nizhny Novgorod, Russia \\ ${ }^{10}$ Lawrence Livermore National Laboratory, Livermore, California 94550, USA
}

(Received 10 November 2014; published 28 July 2015)

\begin{abstract}
The production of neutron beams having short temporal duration is studied using ultraintense laser pulses. Laser-accelerated protons are spectrally filtered using a laser-triggered microlens to produce a short duration neutron pulse via nuclear reactions induced in a converter material $(\mathrm{LiF})$. This produces a $\sim 3 \mathrm{~ns}$ duration neutron pulse with $10^{4} \mathrm{n} / \mathrm{MeV} / \mathrm{sr} / \mathrm{shot}$ at $0.56 \mathrm{~m}$ from the laser-irradiated proton source. The large spatial separation between the neutron production and the proton source allows for shielding from the copious and undesirable radiation resulting from the laser-plasma interaction. This neutron pulse compares favorably to the duration of conventional accelerator sources and should scale up with, present and future, higher energy laser facilities to produce brighter and shorter neutron beams for ultrafast probing of dense materials.
\end{abstract}

DOI: 10.1103/PhysRevLett.115.054802

Because of their direct interaction with nuclei, neutrons penetrate deep into materials and can probe ion population properties directly. This leads to unique insights into many aspects of matter beyond the capability of charged particles or $\mathrm{x}$ rays. However, as neutron probing techniques advance, a challenge is to create a source that is short in time for the observation of ultrafast (picosecond to nanosecond) phenomena. For neutron energies in the $\mathrm{MeV}$ range, neutron damage can be understood at the atomic scale: creating collision cascades, point defects, dislocation, or melts that could be observed in real time [1]. This is a highly topical subject for many technological applications: radiation hardness testing of semiconductors in aerospace, materials for fusion or fission reactors [2-5], particle accelerator vessels, or containers for storing radioactive nuclear wastes. For slightly lower $(\mathrm{keV})$ energies, understanding of the dynamic behavior of warm dense matter (WDM) [6] is a key application area for short neutron bursts $[7,8]$. As neutrons are uniquely sensitive to ion properties, such a source would allow (e.g., via resonance spectroscopy [7]) measurement of ion distribution temperatures when synchronized with a WDM-driving laser or free-electron laser source, which have a short (femtosecond-nanosecond) temporal duration.

Subpicosecond duration lasers are superb candidates for the production of short-pulse neutron probes. In fact, such high-intensity lasers have previously produced monoenergetic neutron bunches of 0.2 ns duration [9]
PACS numbers: 29.25.Dz, 52.38.Kd, 52.58.Lq, 52.38.Ph

via irradiation of deuterium clusters (D-D reactions). However, this method is limited to monoenergetic neutrons and has a low neutron yield $\left(\sim 10^{5} \mathrm{n} / \mathrm{J}\right)$ compared to methods based on laser-accelerated protons impinging on solid material slabs, a technique shown to create up to and above $10^{8} \mathrm{n} / \mathrm{sr} / \mathrm{shot}\left(\sim 10^{7} \mathrm{n} / \mathrm{J}\right)[5,8,10-13]$. On the other hand, a major challenge when using solid targets is that the interaction creates large numbers of highenergy charged particles and $x$ rays, which could damage a test sample and create a high-noise environment for sensitive diagnostics. Also, while picosecond duration at the source, the laser-accelerated protons are broadband in energy and divergent in angle; thus, both their temporal cohesion and flux decrease rapidly over large distances. While techniques exist to focus and select ions via pulsed or passive magnetic fields [14-17], these devices require large distances to function, which leads to increased timeof-flight broadening and takes up valuable space for the shielding of unwanted particles.

In this Letter, we show, by modifying the proton spectrum, that a high-flux, short duration, and narrow band laser-generated neutron source can be produced far from the secondary radiation generated in the laser-interaction using laser techniques alone. This is achieved using a laserdriven microlens [18-20], to both transport the protons and narrow their energy spread and temporal duration, which results in a neutron source that is similarly improved. This method is shown to produce a neutron bunch with a $\sim 3 \mathrm{~ns}$ 


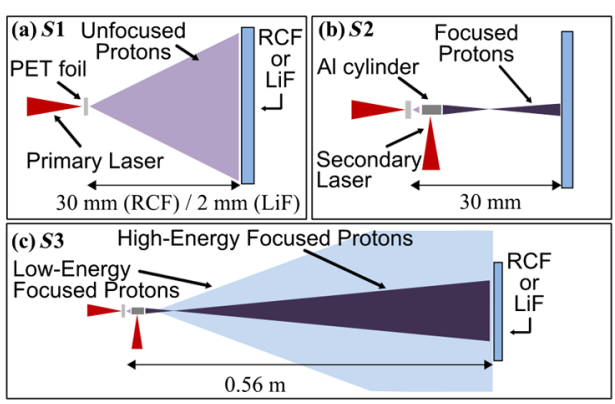

FIG. 1 (color online). Diagrams of the different setups used in this study: (a) $S 1$ : the broadband, unfocused proton beam, (b) $S 2$ : proton focusing using the microlens, irradiated by the secondary beam, and (c) $S 3$ : focused protons at longer distances, where the higher divergence of the lower-energy protons decreases their contribution to the measured spectrum.

full-width-at-half-maximum (FWHM) duration at $0.56 \mathrm{~m}$ from the primary target, thus allowing the neutrons to be produced and used in a region free of high-energy secondary particles.

This study was preformed in three stages, as illustrated in Fig. 1. Stage I $(S 1)$ accelerated protons from the primary $50 \mu \mathrm{m}$ solid polyethylene terephthalate plastic target (coated with $14 \mathrm{~nm} \mathrm{Al}$ to improve laser absorption) using the main beam of the ELFIE laser [21] (10 J, 350 fs pulse length, $10 \mu \mathrm{m}$ focal spot, $1057 \mathrm{~nm}$ wavelength, 20 min shot rate) located at the Laboratoire pour l'Utilisation des Lasers Intenses (LULI) in France. The laser accelerates electrons which generate a strong electric field (MV potential) and accelerate protons via the target normal sheath acceleration (TNSA) mechanism [22-25]. The protons generally have broadband energy spectrum and divergence half angles of $5-30^{\circ}[26,27]$.

In stage II $(S 2)$, the protons were chromatically focused using a plasma-based microlens $[18,19]$. The hollow Al cylinder $(\phi=0.86 \mathrm{~mm}$, thickness $=0.10 \mathrm{~mm}$, length $=3.0 \mathrm{~mm}$ ) used as a microlens was placed $1.0 \mathrm{~mm}$ from the proton source and irradiated with the secondary ELFIE laser beam (similar parameters to the main beam) at a time delay of $86 \mathrm{ps}$ following the primary pulse. The secondary laser pulse creates a strong transient electric field (as in TNSA) normal to the cylinder surface, which forces the protons towards the cylinder axis. Lowerenergy protons spend more time in the field; thus, they are focused more strongly and subsequently have a higher divergence, $8^{\circ}$, compared to higher energy protons, which are focused less and obtain a lower divergence, $1^{\circ}$. The timing of the secondary laser is important, as this determines the maximum energy of protons that will be focused by the cylinder since protons arriving prior to this time will feel no effects of the field.

In stage III (S3) the chromatic nature of the focus is used to reduce the fluence of lower-energy protons arriving at a distance of $0.56 \mathrm{~m}$ from the proton source. Since the lower energy protons are more divergent (as the associated focal length of the microlens is shorter for them $[18,19])$, they become more diluted than the higher energy protons [see Fig. 1(c)], which increases the ratio of the high-tolow energy protons. At this point, in the center of the beam the energy bandwidth — and, thus, the temporal duration of the proton beam-is narrowed compared to the intrinsic beam and can be used to create a short-pulse neutron beam through nuclear reactions.

In our discussion of the experimental results, we begin with the diagnosis of the proton beam. Both the proton beam widths and the spectra were measured using layers of Gafchromic ${ }^{\circledR}$ Radiochromic Film (RCF) [26]. In the $S 1$ and $S 2$ cases, both HD and MD films were used to increase the dynamic range of the measurement, while in the $S 3$ case only the MD film (more sensitive, but lower energy resolving) was used due to the lower proton fluence at this distance.

Figure 2(a) shows the protons in the unfocused $(S 1)$ case using RCF placed at $30 \mathrm{~mm}$. These protons have a divergence angle that decreases with increasing proton energy, as visible in the diameters ( $35 \mathrm{~mm}$ at $1.3 \mathrm{MeV}$ and $6 \mathrm{~mm}$ at $8.9 \mathrm{MeV}$ ). The next set of images [Fig. 2(b)] shows the focused protons $30 \mathrm{~mm}$ from the target $(S 2)$. Here, the beam diameter is significantly reduced and the diameter decreases as a function of energy $(8 \mathrm{~mm}$ at $1.3 \mathrm{MeV}$ and $2 \mathrm{~mm}$ at $7.7 \mathrm{MeV}$ ) due to the focusing effect discussed previously. However, protons with energies above $7.7 \mathrm{MeV}$ transit the cylinder before the electric field has developed and are not focused; thus, their shape represents the projection of the cylinder aperture.

Finally, in Fig. 2(c), the focused beam is shown at $0.56 \mathrm{~m}$ (S3) from the target. The beam is not completely centered on the film (by an offset of $1.8^{\circ}$ ), and thus we capture around a quarter of the proton beam. Also, we note that the beam appears more annular at this larger distance. The diameter is still energy dependent $(67 \mathrm{~mm}$ at $3.8 \mathrm{MeV}$ and $34 \mathrm{~mm}$ at $6.3 \mathrm{MeV}$ ) and corresponds well to the expected diameter using simple trigonometric ratios of the focal

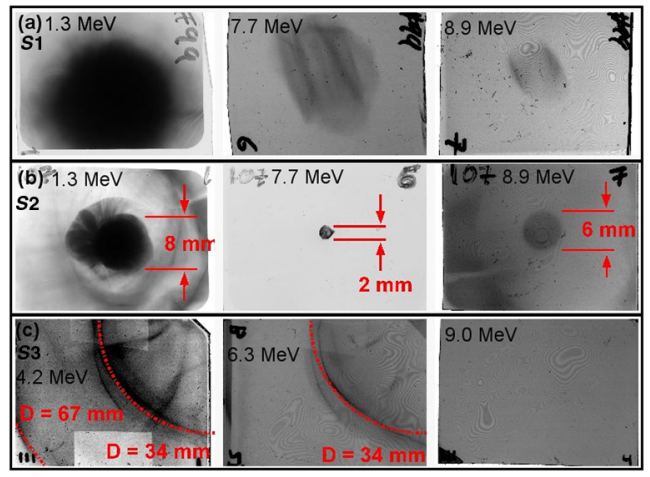

FIG. 2 (color online). Images of RCF for (a) unfocused protons in the $S 1(30 \mathrm{~mm})$ position, (b) focused protons in the $S 2(30 \mathrm{~mm})$ position, and (c) focused protons in the $S 3(0.56 \mathrm{~m})$ position. The energies marked on the films correspond to the average proton energy incident on the layer. All films are plotted with the same spatial scale, but they have different gray scales. 


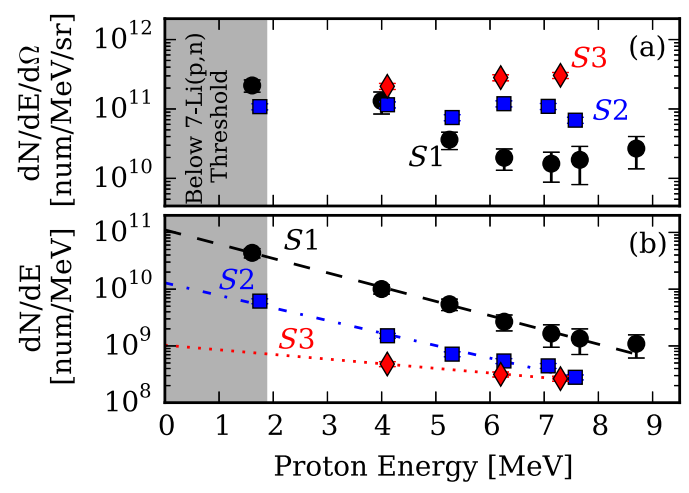

FIG. 3 (color online). Measured single-shot energy spectra of the proton fluence from $S 1$ (the circles), $S 2$ (the squares), and $S 3$ (the diamonds). Error bars for $S 1$ represent variation across multiple shots, whereas $S 2$ and $S 3$ are single shots with $10 \%$ film batch variability uncertainty. (a) The average fluence, which is determined by dividing the total signal on the film by the solid angle subtended by the signal. (b) The total number of protons hitting the film stack, where lines show exponential fits to the spectra, $d N / d \mathcal{E}=(N / T) e^{-\mathcal{E} / T}$. Where $N$ is $1.9 \times 10^{11}$, $2.5 \times 10^{10}$, and $5.5 \times 10^{9}$, and $T$ is $1.72,1.96$, and $5.32 \mathrm{MeV}$ for the $S 1, S 2$, and $S 3$ cases, respectively.

distances measured in the $S 2$ setup ( $81 \mathrm{~mm}$ at $3.7 \mathrm{MeV}$ and $50 \mathrm{~mm}$ at $6.1 \mathrm{MeV}$ ).

Figure 3(a) shows the energy spectra of the proton fluence inferred from the RCF stacks using the unfolding method described in Ref. [28], Sec. 3.1. The intrinsic spectrum $(S 1)$ decreases monotonically and shows a decrease in proton number by a factor of 100 between 1.3 and $6 \mathrm{MeV}$. When the protons are focused $(S 2, S 3)$, the spectrum changes considerably. Instead of decreasing monotonically, the spectrum has a second peak at around $6 \mathrm{MeV}$, where the proton number is ten times higher than the intrinsic spectrum at this energy and is relatively flat from 4 to $8 \mathrm{MeV}$. At the high energies, protons are less divergent than the lower-energy ones and thus produce a higher fluence at long distances. Since we will use a small $\mathrm{LiF}$ neutron converter at this location, only protons in the central portion of the beam will contribute to neutron production [illustrated in Fig. 1(c)]. This takes advantage of this increase in high-to-low energy protons to narrow the energy and temporal duration of the neutron bunch. Figure 3(b) shows exponential fits to the proton spectra that are used later as inputs for neutron generation simulations. These fits show that the focusing technique has an efficiency of around $15 \%$, which is the efficiency that we expect from the geometry of the Al cylinder and the intrinsic proton divergence, and could thus be increased by using a wider diameter cylinder.

Neutrons were produced by placing a $\mathrm{LiF}$ disk $(\phi=25 \mathrm{~mm}$, thickness $=2 \mathrm{~mm})$ in the path of the proton beam at $2 \mathrm{~mm}$ and $0.56 \mathrm{~m}$ away in the $S 1$ and $S 3$ cases, respectively. The protons produce neutrons via nuclear reactions in the LiF, mainly ${ }^{7} \mathrm{Li}(p, n)$. The neutron yield at $0^{\circ}$ was diagnosed using CR-39, a plastic damaged indirectly by neutrons ( $\sim 0.5$ to $6.0 \mathrm{MeV})$ through scattered ions [29], and bubble detectors, a supercritical solution that creates bubbles when neutrons (1.0 to $2.5 \mathrm{MeV})$ scatter in the solution [30,31]. The CR-39 detectors and bubble detectors were placed 20 and $40 \mathrm{~mm}$ from the LiF disk, respectively. To improve the statistics, they were integrated over three to four shots. The CR-39 was shielded from direct protons $(<21 \mathrm{MeV})$ with $1 \mathrm{~mm}$ of $\mathrm{Pb}$. The CR-39 and bubble detectors recorded yields of $7 \pm 4$ and $8 \pm 4 \times 10^{5} \mathrm{n} / \mathrm{MeV} / \mathrm{sr} /$ shot, respectively, in the $S 1$ case. We note that these yields are lower than those presently achievable on some laser systems due to the higher laser intensity and contrast of such systems [5,8,10-13].

To measure the spectrum and temporal duration of the neutron bunch, a neutron time-of-flight (ntof) detector was employed. The ntof used a $40 \times 40 \times 120 \mathrm{~mm}$ BC400 scintillator (placed so neutrons traverse the $40 \mathrm{~mm}$ length) coupled to a Photonis XP2972 photomultiplier and recorded with a $1 \mathrm{GHz}$ oscilloscope. This was placed at $2.2 \mathrm{~m}$ and $30^{\circ}$ from the $S 1 \mathrm{LiF}$ position, which corresponds to $2.0 \mathrm{~m}$ and $33^{\circ}$ from the $S 3 \mathrm{LiF}$ position. The ntof was shielded with $200 \mathrm{~mm}$ of $\mathrm{Pb}$ to reduce the $\mathrm{X}$-ray signal produced in the scintillator.

Figure 4 shows a comparison between the ntof signals without $(S 1)$ and with $(S 3)$ spectral modification of the incident protons. The light-colored lines show experimental results when no neutrons are expected (i.e., RCF was used instead of LiF). The earliest signal, at $\sim 10$ ns (the peak is not visible due to the scale), is due to $x$ rays induced by electron bremsstrahlung in the primary target, with decays based on the response time of the scintillator, and is used to synchronize to the time zero of the laser. We show this non-neutron case to highlight the clean exponential decay of the scintillator signal (i.e., there are no reflections or spurious signals).

The dark lines in Fig. 4 show the experimental signal recorded when using $\mathrm{LiF}$ for neutron production. The upper axis (neutron energy) is derived from the location of the

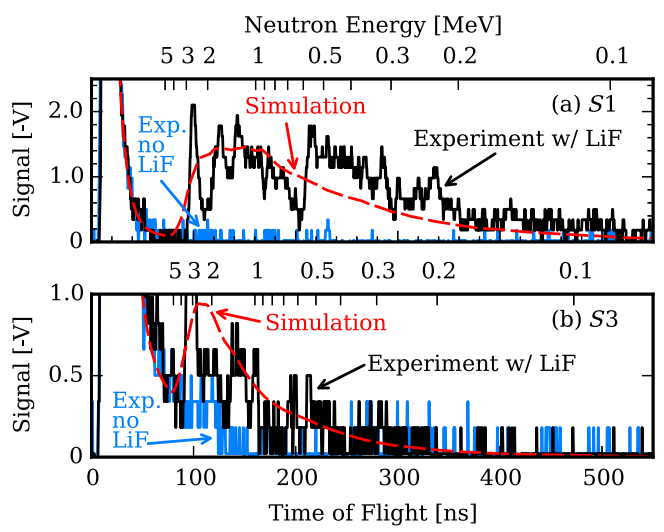

FIG. 4 (color online). Plot of the ntof traces from the experimental data without neutron production (the light lines), with neutron production (the dark lines), and the simulated neutron signal (the dashed lines). Time zero corresponds to the arrival of the laser. Panels (a) and (b) refer to setups $S 1$ and $S 3$, respectively. 
ntof with respect to the $\mathrm{LiF}$ (taking into account the flight time of the protons in the $S 3$ case). We see that the first neutrons arrive around $100 \mathrm{~ns}$, corresponding to an energy of $\sim 3 \mathrm{MeV}$. In the $S 1$ case, the neutron signal extends many hundreds of nanoseconds in time and with energies down to $100 \mathrm{keV}$. In contrast, the $S 3$ neutron signal is much shorter in duration and has a significant reduction of lower-energy neutrons due to the modified incident proton spectrum.

The narrow peak in the signal (at $\sim 100 \mathrm{~ns}$, with a peak of $2.0 \mathrm{~V}$ - thus out of the graph) in $S 3$ is likely due to a single neutron hitting the scintillator. This is because, at this energy $(3 \mathrm{MeV})$, the scattering probability is only $50 \%$ in our scintillator and the neutron flux on the detector is low, only $\sim 0.1 \mathrm{n} / \mathrm{ns}$. This explanation is corroborated by the observed similarity between this narrow pulse and the detector's temporal response (both 9 ns FWHM). We note that this statistically limited feature is less important as energy decreases since the average scattering probability is already $90 \%$ at $1 \mathrm{MeV}$.

In order to better understand these signals, the Monte Carlo particle transport code MCNP6 [32] was run. This code includes particle scattering, energy loss, and nuclear interactions. The proton-induced nuclear reactions included were ${ }^{7} \mathrm{Li}(p, n)^{7} \mathrm{Be},{ }^{6} \mathrm{Li}(p, n){ }^{6} \mathrm{Be}$ and ${ }^{19} \mathrm{~F}(p, n){ }^{19} \mathrm{Ne}$, which have cross sections resolved in angle. The simulation did not include reactions producing excited states in the final nuclei or other reaction channels yielding neutrons such as breakup; however, these have cross sections below $10 \%$ of the main reactions. When benchmarking the code, we noticed that the standard ${ }^{7} \operatorname{Li}(p, n)$ cross section (ENDF/B-VII.0 [33]) used by the code differed considerably from the experimental data [34], as does TENDL-2013 [35] below $15 \mathrm{MeV}$. Thus, a recently reworked cross section was used instead [36].

The geometry of the simulation reproduced the experimental setup with protons injected from the location of the primary target and directed, using straight trajectories, towards a $\mathrm{LiF}$ disk at the $S 1$ or $S 3$ position. The protons were given the measured exponential spectra described in Fig. 3(b). For the $S 1$ case, our simulations recorded a neutron yield in the forward direction of 2.8 and $3.9 \times 10^{5} \mathrm{n} / \mathrm{MeV} / \mathrm{sr} /$ shot in the energy range of the CR39 and bubble detectors, respectively, which is consistent with the experimental measurements.

To reproduce the neutron time-of-flight data, detectors were placed in the simulations at the same location and dimensions as the scintillators used in the experiment. The simulations included the neutron scattering induced from $\mathrm{Pb}$ shielding along the line of sight from source to detector, as well as scattering sources near the detector (e.g., the steel chamber, concrete walls, concrete floor, and other $\mathrm{Pb}$ walls). MCNP6 collected the number of neutrons passing through the detector and binned them in energy and time. The neutron detector efficiency was determined in the following manner. First, the spectrum of protons scattered via neutrons (at these energies, scattering of $\mathrm{C}$ and nuclear processes can be neglected) was determined using other simulations with MCNP6. Next, the light response (in units of electron-equivalent response) of the protons was determined by applying the light response function from Birks' formula [37] ( $k B$ value from Ref. [37] for NE-102). Finally, the electron equivalent response was converted to a signal using a calibration performed with a ${ }^{60} \mathrm{Co}$ gamma source to complete the absolute calibration. Additionally, the ${ }^{60} \mathrm{Co}$ source was used to fit the detector's temporal response with two exponential decays $(1 / e=8,60 \mathrm{~ns})$, which was then convolved with the calibrated neutron signal to give a simulated signal, as well as an x-ray falloff.

The simulated signals of the simulations are shown as dashed lines in Fig. 4. These are in agreement with the experimental shape of the data and, especially, the absolute number, which has not been arbitrarily normalized. This agreement highlights the importance of including nearby structures, as we found that these scattering structures (i.e., those not along the direct line of sight) contributed from $40 \%$ to $80 \%$ of the neutrons detected at $3 \mathrm{MeV}$ in the $S 1$ and $S 3$ cases, respectively.

To determine the loss of neutrons through the selection process, we look at the yield of $3.0 \mathrm{MeV}$ neutrons on the scintillator, which are 4.0 and $1.2 \times 10^{4} \mathrm{n} / \mathrm{MeV} / \mathrm{sr} / \mathrm{shot}$ for the $S 1$ and $S 3$ cases, respectively. This shows that the neutron yield was only reduced to $30 \%$ of the original yield, despite the large $(0.56 \mathrm{~m})$ distance traveled by the protons. As mentioned previously, this reduction is due to the geometry of the microlens. Using the simulations, we investigate the temporal narrowing achieved by placing a virtual detector consisting of a neutron counting sphere of $2.5 \mathrm{~mm}$ radius at $0.5 \mathrm{~m}$ from the proton emission (i.e., $5 \mathrm{~mm}$ from the $\mathrm{LiF}$ in the $S 3$ case) to record all of the neutrons that pass through it. This measurement shows a FWHM duration of $30 \mathrm{~ns}$ in the $S 1$ case and $3.4 \mathrm{~ns}$ in the $S 3$ case-thus, a factor of around 10 in temporal narrowing. This reduction is due to the energy selection of the protons hitting the LiF. Since the low-energy protons are dramatically reduced (see Fig. 3) the time-of-flight broadening is reduced as well. This duration compares well with existing accelerator driven devices (Ref. [38] and the references therein). Additionally, we note that moving the LiF slab to $100 \mathrm{~mm}$ would lower the pulse to subnanosecond duration, while at the same time staying a long distance away from the laser interaction to allow for proper shielding.

In summary, we have shown that a narrowing of the pulse duration of protons and neutrons generated by laser acceleration is achievable, showing that laser-accelerated neutrons can be useful in researching and probing material properties at ultrafast time scales. The ability of the microlens to focus ions is expected to scale with the electron temperature (and thus the laser intensity) [39] and has previously been shown via simulations to focus proton beams up to $270 \mathrm{MeV}$ [40], which will be important when using higher laser energies (exceeding $1 \mathrm{~kJ}$, instead of $10 \mathrm{~J}$ ) 
and emerging ion-acceleration mechanisms to give rise to much higher neutron yields [13]. Additionally, the upcoming increase in repetition rate of these lasers (now a few shots per hour) will soon increase with the development of fiber [41] or diode-pumped lasers [42,43]. The smooth electric field profiles [19] and the simple structure (potentially a single target [44]) of the microlens make this technique compatible with quick, repeatable lasers making laser-based short-pulse neutron sources even more attractive.

We are grateful to Dr. K. Parsons and Dr. G. Hale for evaluating a newer ${ }^{7} \mathrm{Li}(p, n)^{7} \mathrm{Be}$ cross section that agrees better with previous experimental results. We acknowledge the support of the LULI technical teams. This work was supported by Grant No. E1127 from Région Île-de-France. The research leading to these results has received funding from Laserlab-Europe (Grant Agreement No. 284464, Ec's seventh framework program) and Grant No. 001528. This work was partly done within the LABEX Plas@Par project and was supported by Grant No. 11-IDEX-0004-02 from Agence Nationale de la Recherche. L. V. was funded by a DGA-MRIS scholarship. H.P., M. M. G., and F. N. acknowledge support from the Romanian National Authority for Scientific Research, CNCS UEFISCDI, under Project No. PN-II-ID-JRP-2011-1. This work also partly supported by the DFG GRK 1203 program and by EPSRC Grants No. EP/K022415/1 and No. EP/J002550/1. M. B. acknowledges cofinancing by the European Social Fund and the state budget of the Czech Republic (Projects No. CZ.1.05/1.1.00/483/02.0061 and No. CZ.1.07/2.3.00/ 20.0279). This work was supported in part by the Ministry of Education and Science of the Russian Federation under Contract No. 14.Z50.31.0007. This work performed under the auspices of the U.S. Department of Energy by Lawrence Livermore National Laboratory under Contract DE-AC52-07NA27344.

[1] R. T. Khaydarov, H. B. Beisinbaeva, M. M. Sabitov, V. Terentev, and G. Berdiyorov, Nucl. Fusion 50, 025024 (2010).

[2] L. J. Perkins, B. G. Logan, M. D. Rosen, M. D. Perry, T. Diaz de la Rubia, N. M. Ghoniem, T. Ditmire, P. T. Springer, and S. C. Wilks, Nucl. Fusion 40, 1 (2000).

[3] J. D. Sethian, A. R. Raffray, J. Latkowski, J. P. Blanchard, L. Snead, T. J. Renk, and S. Sharafat, J. Nucl. Mater. 347, 161 (2005).

[4] U. Fischer, M. Avrigeanu, P. Pereslavtsev, S. P. Simakov, and I. Schmuck, J. Nucl. Mater. 367-370, 1531 (2007).

[5] D. P. Higginson et al., Phys. Plasmas 18, 100703 (2011).

[6] R. P. Drake, High-Energy-Density Physics (Springer, New York, 2006).

[7] V. W. Yuan, J. D. Bowman, D. J. Funk, G. L. Morgan, R. L. Rabie, C.E. Ragan, J.P. Quintana, and H. L. Stacy, Phys. Rev. Lett. 94, 125504 (2005); D. C. Swift, A. Seifter, D. B. Holtkamp, V. W. Yuan, D. Bowman, and D. A. Clark, Phys. Rev. B 77, 092102 (2008).

[8] D. P. Higginson et al., Phys. Plasmas 17, 100701 (2010).
[9] T. Ditmire, J. Zweiback, V.P. Yanovsky, T. E. Cowan, G. Hays, and K. B. Wharto, Nature (London) 398, 489 (1999).

[10] K. L. Lancaster et al., Phys. Plasmas 11, 3404 (2004).

[11] L. Willingale et al., Phys. Plasmas 18, 083106 (2011).

[12] C. Zulick et al., Appl. Phys. Lett. 102, 124101 (2013).

[13] M. Roth et al., Phys. Rev. Lett. 110, 044802 (2013).

[14] S. Ter-Avetisyan, M. Schnürer, R. Polster, P. Nickles, and W. Sandner, Laser Part. Beams 26, 637 (2008).

[15] T. Burris-Mog et al., Phys. Rev. ST Accel. Beams 14, 121301 (2011).

[16] S. Sinigardi, G. Turchetti, F. Rossi, P. Londrillo, D. Giove, C. De Martinis, and P. R. Bolton, Nucl. Instrum. Methods Phys. Res., Sect. A 740, 99 (2014).

[17] S. N. Chen, M. Gauthier, D. P. Higginson, S. Dorard, F. Mangia, R. Riquier, S. Atzeni, J.-R. Marquès, and J. Fuchs, Rev. Sci. Instrum. 85, 043504 (2014).

[18] T. Toncian et al., Science 312, 410 (2006).

[19] T. Toncian et al., AIP Adv. 1, 022142 (2011).

[20] C. Ellison and J. Fuchs, Phys. Plasmas 17, 113105 (2010).

[21] J. P. Zou, C. L. Blanc, P. Audebert, S. Janicot, A. M. Sautivet, L. Martin, C. Sauteret, J. L. Paillard, S. Jacquemot, and F. Amiranoff, J. Phys. Conf. Ser. 112, 032021 (2008).

[22] A. P. Fews, P. A. Norreys, F. N. Beg, A. R. Bell, A. E. Dangor, C. N. Danson, P. Lee, and S. J. Rose, Phys. Rev. Lett. 73, 1801 (1994).

[23] R. A. Snavely et al., Phys. Rev. Lett. 85, 2945 (2000).

[24] S. C. Wilks, A. B. Langdon, T. E. Cowan, M. Roth, M. Singh, S. Hatchett, M. H. Key, D. Pennington, A. MacKinnon, and R. A. Snavely, Phys. Plasmas 8, 542 (2001).

[25] J. Fuchs et al., Nat. Phys. 2, 48 (2006).

[26] A. Mančić, J. Fuchs, P. Antici, S. Gaillard, and P. Audebert, Rev. Sci. Instrum. 79, 073301 (2008).

[27] P. Bolton et al., Phys. Med. 30, 255 (2014).

[28] D. P. Higginson et al., High Energy Density Phys., doi: 10.1016/j.hedp.2014.11.007 (2014).

[29] J. Frenje et al., Rev. Sci. Instrum. 73, 2597 (2002).

[30] Bubble Technology Industries Inc., http://www.bubbletech .ca.

[31] A. Green, S. Kar, and H. Powell, in Central Laser Facility Annual Report, 2012-2013.

[32] D. Pelowitz, MCNP6 User's Manual, Version 1, Report No. LA-CP-13-00634, Los Alamos National Laboratory (2013).

[33] M. B. Chadwick et al., Nucl. Data Sheets 107, 2931 (2006).

[34] H. Liskien and A. Paulsen, At. Data Nucl. Data Tables 15, 57 (1975); S. Abramovich et al., J. Vop. At. Nauki i Tekhn., Ser. Yadernye Konstanty 4, 17 (1984).

[35] A. J. Koning and D. Rochman, Nucl. Data Sheets 113, 2841 (2012).

[36] K. Parsons and G. Hale (private communication).

[37] R. Craun and D. Smith, Nucl. Instrum. Methods 80, 239 (1970).

[38] E. Altstadt et al., Ann. Nucl. Energy 34, 36 (2007).

[39] S. Gordienko, T. Baeva, and A. Pukhov, Phys. Plasmas 13, 063103 (2006).

[40] E. d'Humières et al., AIP Conf. Proc. 877, 41 (2006).

[41] G. Mourou, B. Brocklesby, T. Tajima, and J. Limpert, Nat. Photonics 7, 258 (2013).

[42] A. Bayramian et al., J. Opt. Soc. Am. B 25, B57 (2008).

[43] T. Gonçalvès-Novo, D. Albach, B. Vincent, M. Arzakantsyan, and J.-C. Chanteloup, Opt. Express 21, 855 (2013).

[44] S. Kar et al., Phys. Rev. Lett. 100, 105004 (2008). 